\title{
ENDEMIC LEPROSY IN NEW YORK CITY
}

\author{
William R. Levis, MD ${ }^{1}$, Lilly-Rose Paraskevas, MD², Mark Jacobson, MD $^{3}$, John Spencer, \\ $\mathbf{P h D}^{4}$, Trudy Spencer, RN, $\mathbf{M P H}{ }^{1}$, and Frank Martiniuk, $\mathbf{P h D}^{5,6}$ \\ ${ }^{1} \mathrm{NYU}$ School of Medicine Department of Dermatology Director of New York Hansen's disease \\ Program, Bellevue Hospital Center, 27 $7^{\text {th }}$ and First Ave., NY, NY 10016
}

2Dermpathdiagnostics, Port Chester, NY 10573

${ }^{3} 520$ Franklin Ave. Garden City, NY

${ }^{4}$ Colorado State University-Department of Microbiology, Immunology and Pathology, Fort Collins, CO 80523

${ }^{5}$ NYU School of Medicine, Department of Medicine-Pulmonary Division, NY, NY 10016

\section{Report of case}

Recently, NYU dermatologists have identified two cases of leprosy in New York that have never been outside the United States. The first case of a person never to travel out of the U.S. was a Caucasian chemist from Queens, NY reported in 2000 (1). A more recent case was a black male from the Bronx, New York in 2008 (2). We now report the third proven identified case of leprosy never out of the continental United States.

The patient is a 48 year-old Black woman complaining of skin discoloration of the face, hands and legs for one year. On exam she was well-developed appearing in no acute distress; eyes and mucous membranes were normal. Over the right and left posterior calf there were two lichenified plaques and surrounding hypopigmentation and dyschromia of the skin with hypoesthesia. Biopsy was compatible with BL (Figure 1A-D). It is important to order a Fite stain when suspecting leprosy as the Ziehl-Neelsen stain is usually weak, as in this case, and often is negative. Her lab results were within normal ranges. ELISA was performed against M. leprae Ag85B (ML2028), ND-O-HSA (PGL-I) and LepLAM and compared to three LL patient sera (LL8, LL27 and LL32) which are very well characterized to have high reactivity to all three antigens by ELISA and Western blot (Figure 2) (3). The titer for this patient's serum is consistent with BL/LL individuals.

\footnotetext{
${ }^{6}$ Corresponding author 212 562-3616; martif02@nyumc.org.

Author Contributions: Dr(s) William R. Levis MD, Lilly-Rose Paraskevas, MD, Mark Jacobson, MD, John Spencer PhD, Trudy Spencer, RN, MPH, and Frank Martiniuk, PhD, had full access to all of the data in the study and take responsibility for the integrity of the data and the accuracy of the data analysis. Study concept and design: $\operatorname{Dr}(\mathrm{s})$ William R. Levis MD, Lilly-Rose Paraskevas, MD, Mark Jacobson, MD, John Spencer PhD, Trudy Spencer, RN, MPH, and Frank Martiniuk, PhD. Acquisition of data: Dr(s) William R. Levis MD, Lilly-Rose Paraskevas, MD, Mark Jacobson, MD, John Spencer PhD, Trudy Spencer, RN, MPH, and Frank Martiniuk, $\mathrm{PhD}$. Analysis and interpretation of data: Dr(s) William R. Levis MD, Lilly-Rose Paraskevas, MD, Mark Jacobson, MD, John Spencer PhD, Trudy Spencer, RN, MPH, and Frank Martiniuk, PhD. Drafting of the manuscript: Dr(s) William R. Levis MD, LillyRose Paraskevas, MD, Mark Jacobson, MD, John Spencer PhD, Trudy Spencer, RN, MPH, and Frank Martiniuk, PhD. Critical revision of the manuscript for important intellectual content: Dr(s) William R. Levis MD, Lilly-Rose Paraskevas, MD, Mark Jacobson, MD, John Spencer PhD, Trudy Spencer, RN, MPH, and Frank Martiniuk, PhD, Obtained funding: Dr(s) William R. Levis MD, Lilly-Rose Paraskevas, MD, Mark Jacobson, MD, John Spencer PhD, Trudy Spencer, RN, MPH, and Frank Martiniuk, PhD.

Financial Disclosure: None reported.
} 


\section{Comment}

Since the immigration act of 1964 there has been a major influx of Hispanic and Asian immigrants into the United States, including regions where leprosy is most prevalent. A 1982 epidemiologic report of leprosy in New York City showed 99 of 100 of the most recent leprosy cases at the former USPHS (United States Public Health System) Hospital on Staten Island, NY were foreign born (4). There were cases of leprosy in foreign born patients who had been in the U.S. for as long as 38 years, raising the question of acquiring the infection in some of NYC's immigrant communities. The first case of leprosy that proved leprosy can be acquired in the Northeastern U.S was a Caucasian chemist from Queens who was never out of the U.S. (2). In that same report, a nurse from N.J. who had gone to China and stayed in first class hotels for a week was reported with leprosy. Leprosy can also masquerade as lupus. A typical lepromatous case from the Dominican Republic was treated as lupus by a rheumatologist with high dose corticosteroids in the same township as our patient, before being recognized as a case of leprosy. Dermatologists often are our first-line sentinels likely to see leprosy. If a case is detected, please contact the HRSA website (www.hrsa.gov/hansens/) and the closest regional Hansen's disease program. Recently, an interaction of M. leprae with HIV has been discovered, usually presenting as immunologic reconstitution inflammatory syndrome (IRIS)(5). This interaction is complex, appears different than the interaction of HIV and TB. TB accelerates the progression of HIV infection to a greater degree than M.leprae. Most importantly, physicians should be aware that leprosy can present as IRIS.

\section{Acknowledgments}

Funding/Support: This study was supported in part by a grant from the CTSI NIH/NCRR 1UL1RR029893-0109. The recombinant protein ML2028(Ag85B) and ND-O-HSA were supplied through the NIH/NIAID Leprosy Research contract NO1 AI-25469 from Colorado State University (www.cvmbs.colostate.edu/mip/leprosy/).

Role of the Sponsors: The sponsors had no role in the design and conduct of the study; in the collection, analysis and interpretation of data; or in the preparation, review or approval of the manuscript.

\section{References}

1. Levis WR, Vides EA, Cabrera A. Leprosy in the eastern United States. JAMA. 2000; 283:10041005. [PubMed: 10697058]

2. Keo T, Martiniuk F, Latkowski J, Cabrera A, Rom W, Levis WR. Molecular origin of endemic leprosy in New York City. Clin Infect Dis. 2008; 46:899-901. [PubMed: 18269333]

3. Zhang J, Chatterjee D, Brennan PJ, Spencer JS, Liav A. A modified synthesis and serological evaluation of neoglycoproteins containing the natural disaccharide of PGL-I from Mycobacterium leprae. Bioorg Med Chem Lett. 2010; 20:3250-3253. [PubMed: 20462755]

4. Levis WR, Schuman JS, Friedman SM, Newfield SA. An epidemiologic evaluation of leprosy in New York City. JAMA. 1982; 247:3221-3226. [PubMed: 7087061]

5. Martiniuk F, Rao SD, Rea TH, Glickman MS, Giovinazzo J, Rom WN, Cabrera A, Levis WR. Leprosy as immune reconstitution inflammatory syndrome in HIV-positive persons. Emerg Infect Dis. 2007; 13:1438-1440. [PubMed: 18252138] 


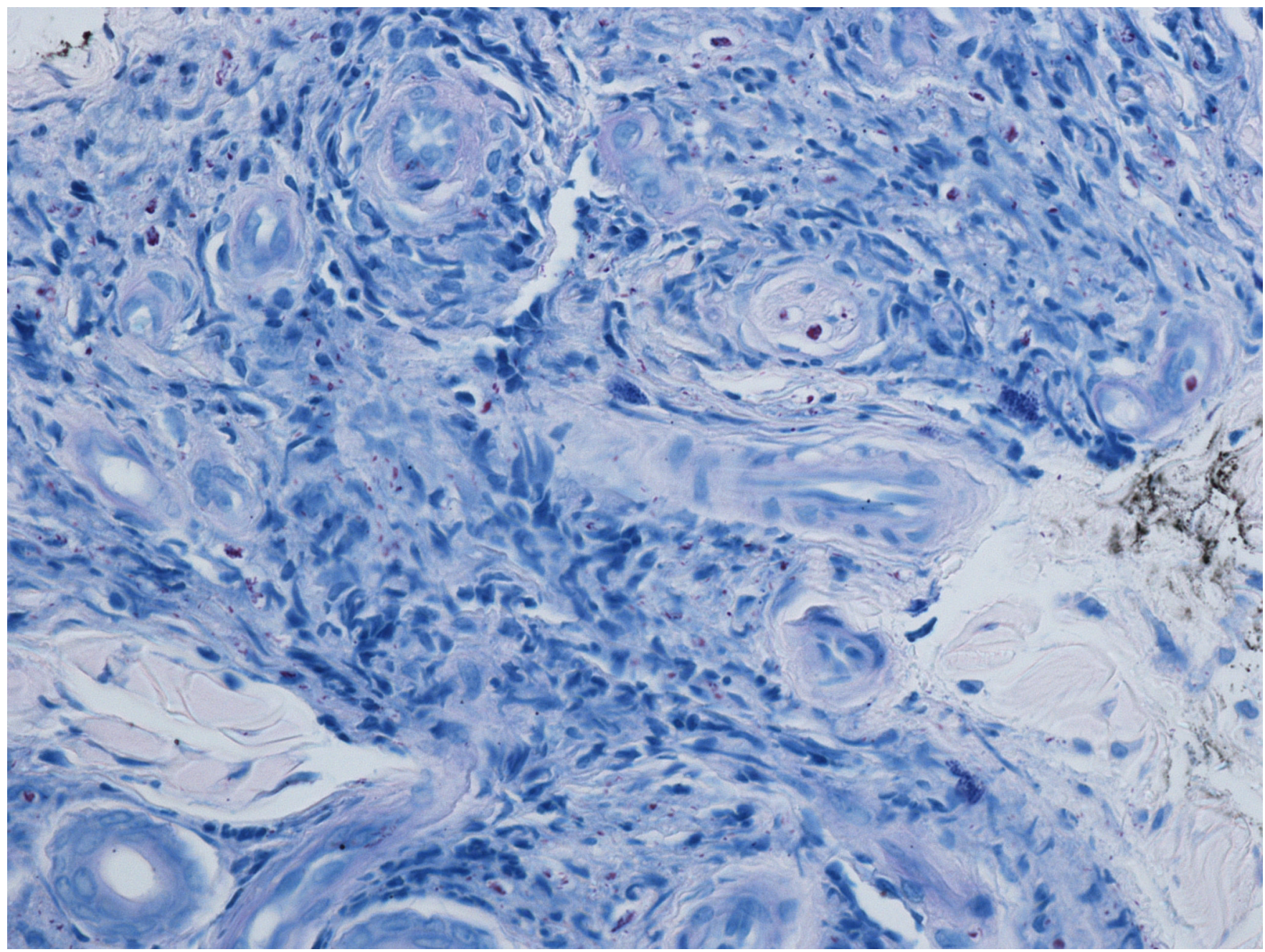




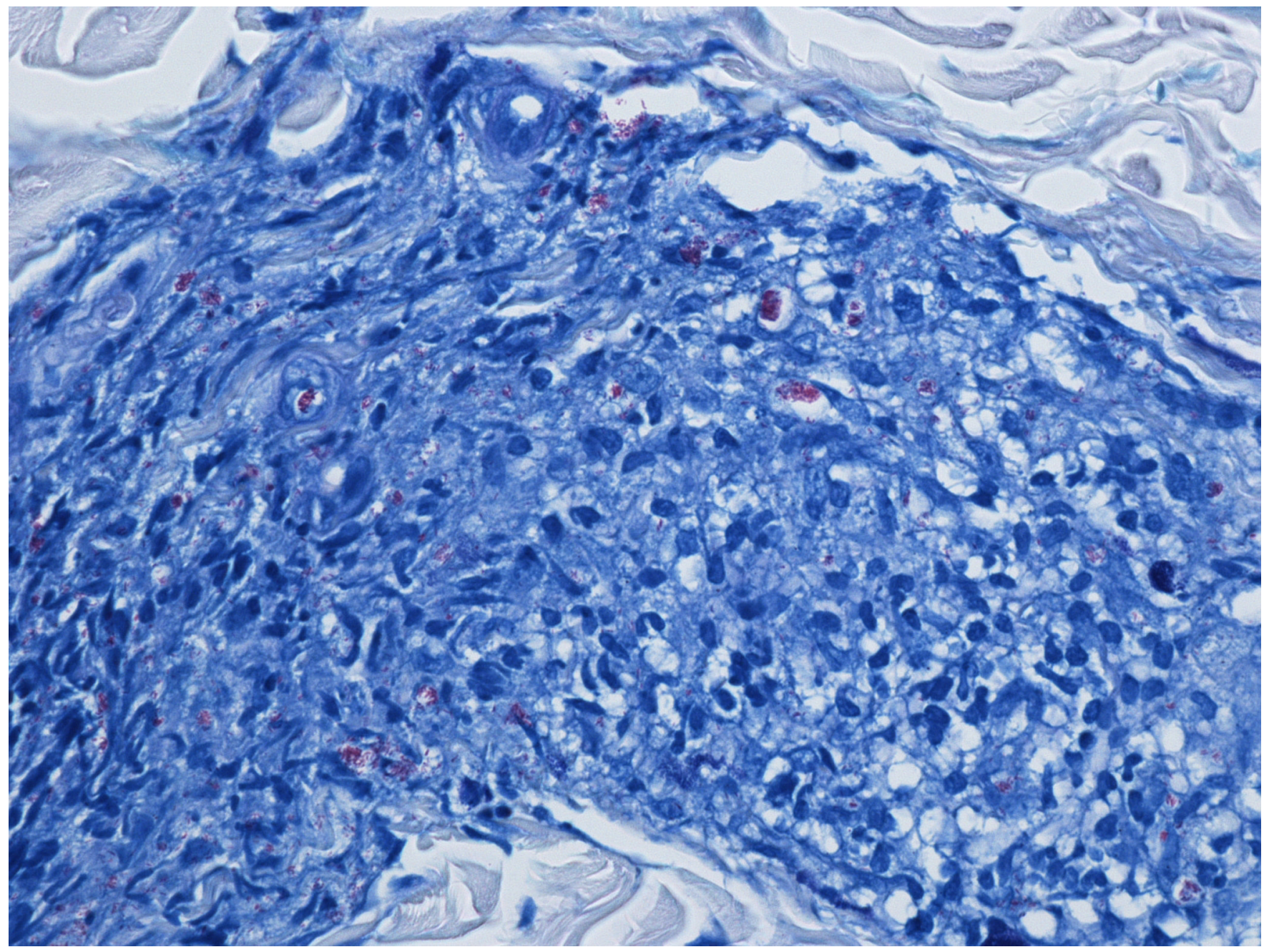

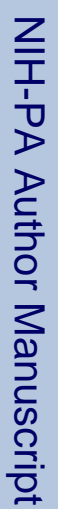




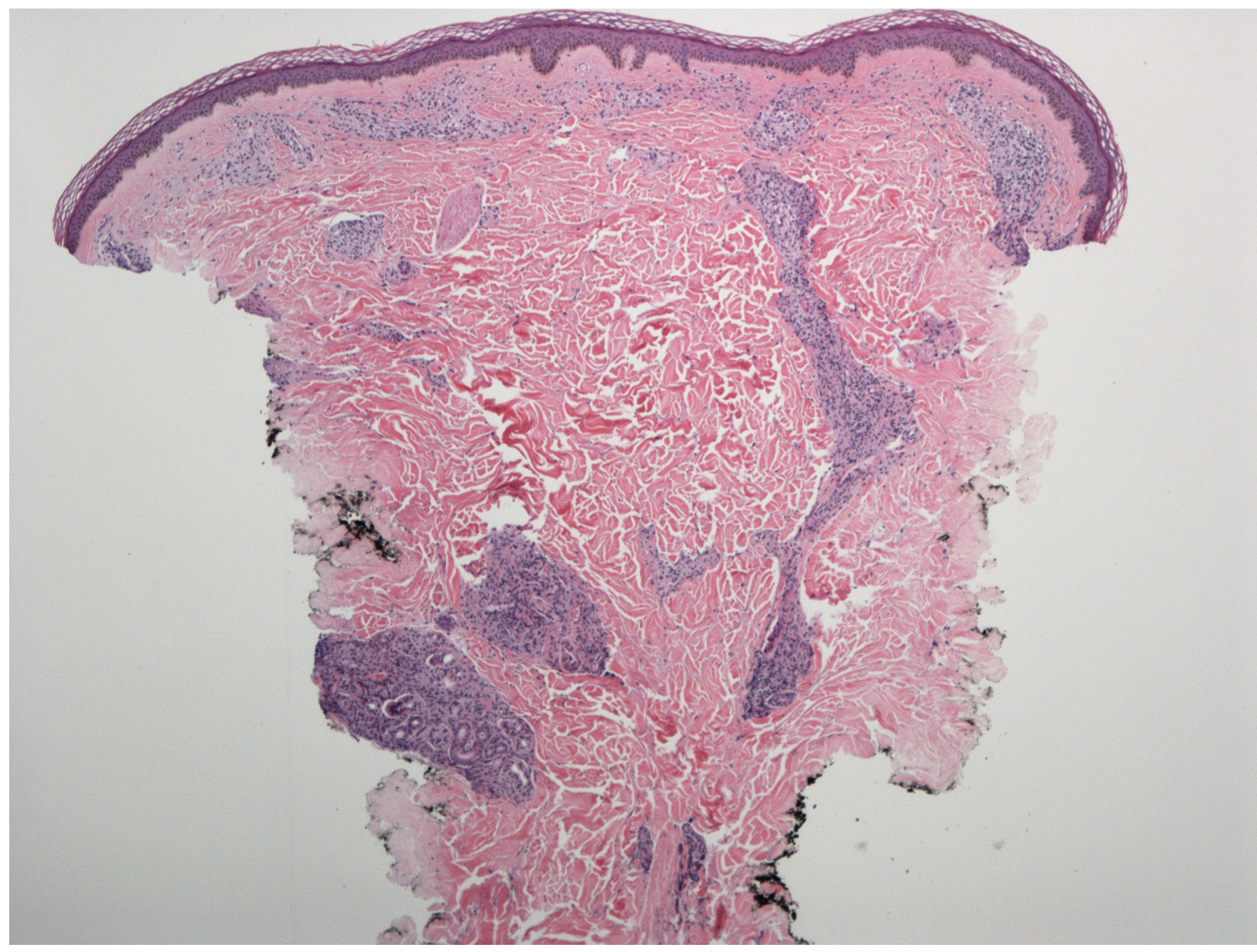




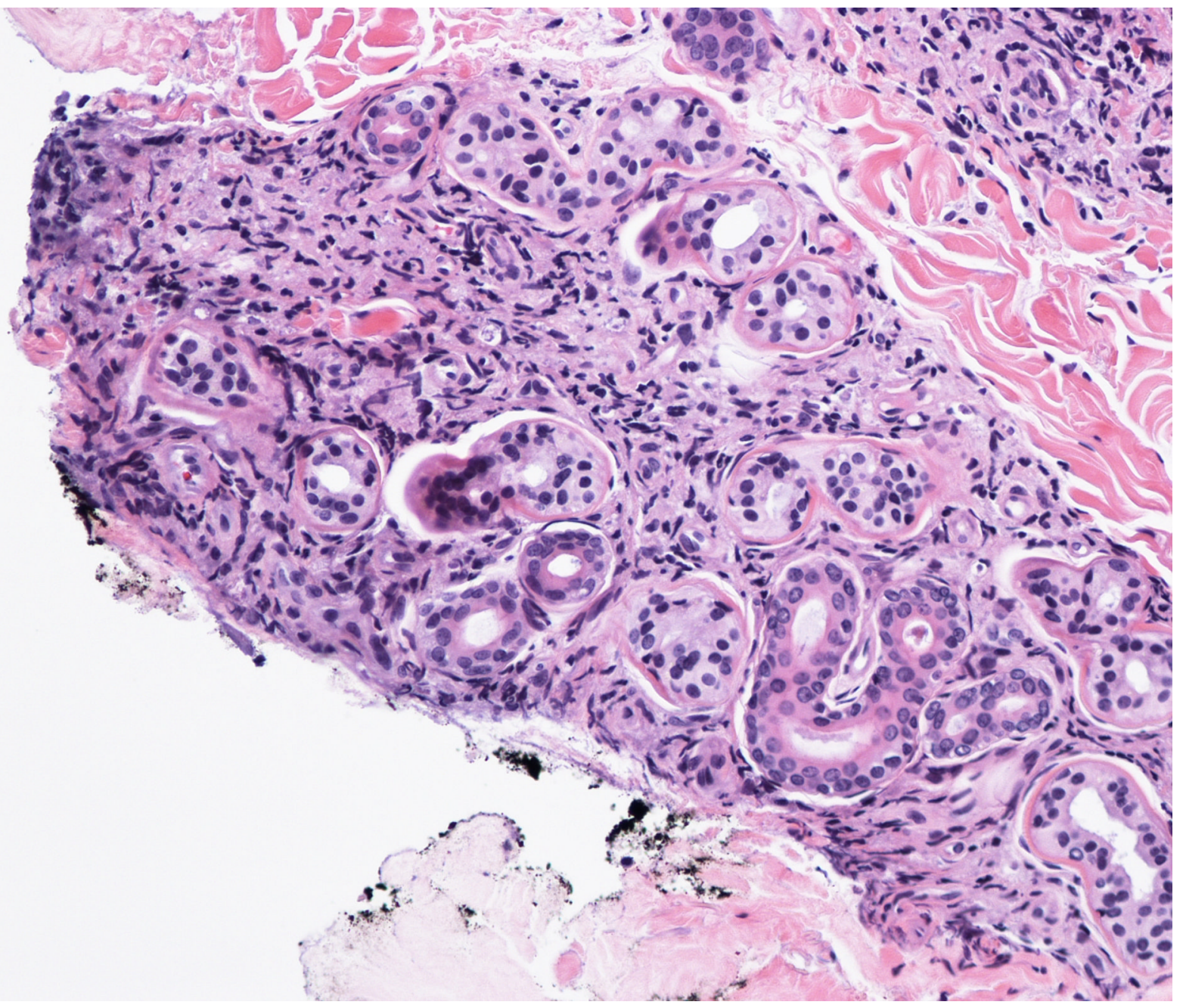

Figure 1.

1A Right lateral biopsy of the calf indicates this case towards the lepromatous pole of leprosy (probably borderline lepromatous). There are histiocytes and collections around blood vessels, nerve and adnexal structures through the dermis. Histiocytes have vacuolated, bluish cytoplasms. Within the cytoplasms there are numerous acid fast bacilli that stain positive with a Fite stain and, to a lesser extent with an AFB stain. PAS and GMS stains are negative. Weak staining of AFB is seen.

1B Fite staining Strong acid fast (in red) Fite staining is seen in many sites.

$1 C \mathrm{H} \& \mathbf{E}$ staining $4 \mathrm{X}$ Low power (4x) of infiltrative dermopathy.

1D H \& E staining 20X High power (20x) shows periadenexal dermal granuloma formation compatible with multi-bacillary leprosy. 

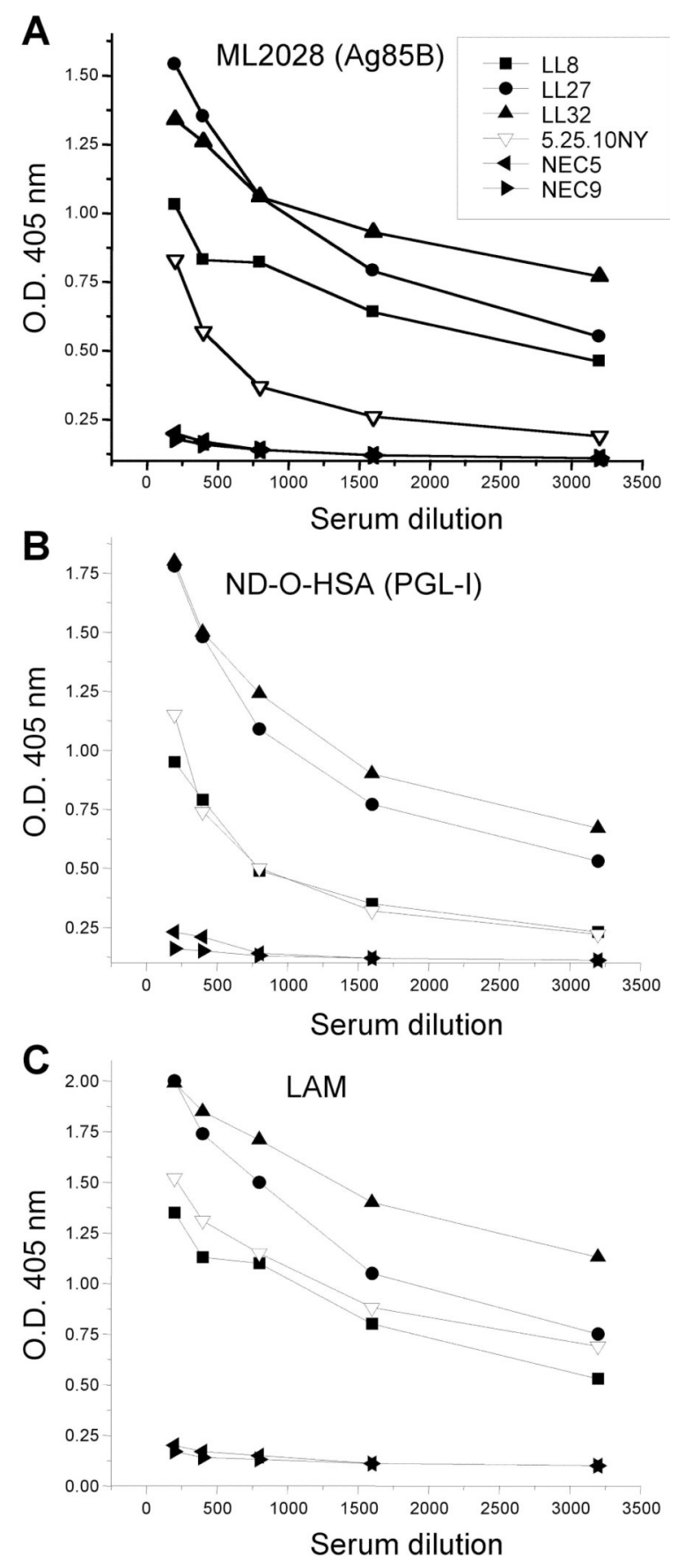

Figure 2.

Reactivity of lepromatous leprosy patient sera as positive controls (LL8, LL27 and LL32) with known high antibody titers to A) recombinant M. leprae protein ML2028 (Ag85B); B) ND-O-HSA (PGL-I); and C) M. leprae lipoarabinomannan (LAM); the patient in this case (5.25.10NY); and non-endemic healthy control sera as negative controls (NEC5, NEC9). The antigens were tested by ELISA using serial two-fold dilutions of serum from 1:200 to 1:3,200. The O.D. readings were determined with a plate reader at A405 nm. 